\title{
Effect of Variation in Test Methods on Performance of Ultraviolet-C Radiation Room Decontamination
}

\author{
Jennifer L. Cadnum, BS; ${ }^{1,2}$ Myreen E. Tomas, MD; ${ }^{1}$ Thriveen Sankar, MNO; ${ }^{1,2}$ Annette Jencson, CIC; ${ }^{1}$ J. Itty Mathew, MLS; ${ }^{2}$ \\ Sirisha Kundrapu, MD; ${ }^{2}$ Curtis J. Donskey, $\mathrm{MD}^{2,3}$
}

ов јестіле. To determine the effect of variation in test methods on performance of an ultraviolet-C (UV-C) room decontamination device. DESIGN. Laboratory evaluation.

METHODs. We compared the efficacy of 2 UV-C room decontamination devices with low pressure mercury gas bulbs. For 1 of the devices, we evaluated the effect of variation in spreading of the inoculum, carrier orientation relative to the device, type of organic load, type of carrier, height of carrier, and uninterrupted versus interrupted exposures on measured UV-C killing of methicillin-resistant Staphylococcus aureus and Clostridium difficile spores.

RESULTS. The 2 UV-C room decontamination devices achieved similar $\log _{10}$ colony-forming unit reductions in the pathogens with exposure times ranging from 5 to 40 minutes. On steel carriers, spreading of the inoculum over a larger surface area significantly enhanced killing of both pathogens, such that a 10 -minute exposure on a $22-\mathrm{mm}^{2}$ disk resulted in greater than $2 \log$ reduction in $C$. difficile spores. Orientation of carriers in parallel rather than perpendicular with the UV-C lamps significantly enhanced killing of both pathogens. Different types of organic load also significantly affected measured organism reductions, whereas type of carrier, variation in carrier height, and interrupted exposure cycles did not.

CONCLUSIONS. Variation in test methods can significantly impact measured reductions in pathogens by UV-C devices during experimental testing. Our findings highlight the need for standardized laboratory methods for testing the efficacy of UV-C devices and for evaluations of the efficacy of short UV-C exposure times in real-world settings.

Infect Control Hosp Epidemiol 2016;37:555-560

Automated ultraviolet-C (UV-C) room decontamination devices are increasingly used as an adjunct to standard cleaning and disinfection in healthcare facilities. ${ }^{1}$ Under experimental conditions, these devices have demonstrated efficacy in killing a variety of bacterial pathogens, including Clostridium difficile spores. $^{2-10}$ In hospital rooms, use of the devices has been shown to reduce the burden of pathogens on surfaces. ${ }^{2-4,6-8,11-13}$ There have also been some reports of use of UV-C devices associated with reductions in healthcare-associated infections. ${ }^{14-16}$

In addition to factors such as cost and ease of use, healthcare facilities considering the purchase of a UV-C room decontamination device must weigh claims regarding the effectiveness of different devices. However, few data are available on the comparative effectiveness of different UV-C devices. ${ }^{5,8}$ Moreover, in contrast to the situation for surface disinfectants, ${ }^{17}$ there are currently no standardized methods recommended for testing of UV-C devices. Thus, a variety of methods have been used in experimental testing. For example, inoculated surfaces have included glass, steel, and Formica carriers or bench countertops, spreading of the inoculum has varied from areas of $4 \mathrm{~mm}^{2}$ to $25 \mathrm{~cm}^{2}$, and inoculated carriers have been oriented in parallel or perpendicular to the bulbs. ${ }^{2-10}$ Although it is known that UV-C efficacy is affected by distance and shading, it is not known if variation in other test conditions affect pathogen killing. Here, we compared the effectiveness of 2 UV-C room decontamination devices and evaluated the effect of variation in test methods on performance of 1 of the devices.

\section{METHODS}

\section{C. difficile and Methicillin-Resistant Staphylococcus aureus} (MRSA) Strains

One strain of each pathogen was studied. The C. difficile strain was American Type Culture Collection (ATCC) strain 43598. Spores were prepared and stored as previously described. ${ }^{18}$ The MRSA strain was a clinical isolate of pulsed-field gel electrophoresis type USA800. MRSA and C. difficile were cultured on selective media as previously described. ${ }^{8}$ 


\section{The UV-C Disinfection Devices}

The Tru-D device (Lumalier) has been described previously. ${ }^{3-5}$ The Clorox Healthcare Optimum-UV System (Clorox) has a 36 inch-diameter circular base and a height of 76 inches. The device is a wheeled unit containing four 64-inch low-pressure mercury lamps emitting 254-nm UV-C light. The device's cycle time is determined by the manufacturer on the basis of room size and configuration. The system contains a motion sensor connected to a safety-rated relay, aborting the cycle if someone enters the room during use.

\section{Comparison of the Optimum-UV and Tru-D Devices for Killing of the Pathogens}

We compared the efficacy of the 2 devices against the pathogens on steel disk carriers in a $10 \times 10$ foot room as described previously. ${ }^{5}$ For each pathogen, $10-\mu \mathrm{L}$ aliquots containing $1 \times 10^{6}$ colony-forming units in phosphate-buffered saline were spread to cover $1-\mathrm{cm}^{2}$ area of stainless steel carriers and allowed to air dry. The carriers were placed perpendicular to the vertical lamps (ie, horizontal) 4 feet from the devices at a height of 4 feet and exposed to cycles of 5, 10, 20, or 40 minutes. For comparison, the Tru-D reflected doses of 12,000 (vegetative cycle) and 22,000 (spore cycle) $\mu \mathrm{Ws} / \mathrm{cm}^{2}$ in single patient rooms require approximately 20 and 45 minutes, respectively. ${ }^{3}$ To quantify viable organisms, treated and untreated control carriers were submersed in $1 \mathrm{~mL}$ of phosphate-buffered saline and vortexed vigorously, and dilutions were plated onto selective media and processed as described previously. ${ }^{3,5,8}$ Because initial experiments demonstrated similar efficacy of the devices, subsequent experiments were conducted only with the Clorox Healthcare Optimum-UV System.

\section{Effect of Inoculum Dispersal on Killing of the Pathogens by the Optimum-UV System}

To assess the impact of dispersal of the inoculum, $10-\mu \mathrm{L}$ aliquots of the pathogens were inoculated onto $10-\mathrm{mm}^{2}$ steel disk carriers (Cole Palmer) either without manual spreading $\left(\sim 4-\mathrm{mm}^{2}\right.$ area $)$ or with spreading $\left(10-\mathrm{mm}^{2}\right.$ area $)$ or onto $22-\mathrm{mm}^{2}$ steel disk carriers with spreading. The carriers were placed perpendicular to the lamps 4 feet from the device at a height of 4 feet in the direct field of radiation and exposed to cycles of 5 or 10 minutes. Reductions were compared with untreated control carriers.

\section{Effect of Orientation of the Carriers Relative to the UV-C Lamps}

We tested the effect of the angle of UV-C incidence by altering the orientation of $22-\mathrm{mm}^{2}$ steel disk carriers relative to the Optimum-UV lamps. The carriers were positioned in parallel with the vertical lamp (ie, vertical orientation directly facing the lamp), perpendicular to the lamp, or at a $45^{\circ}$ angle from the lamp. The carriers were placed 4 feet from the device at a height of 4 feet and exposed to 10 minutes of UV-C.

\section{Effect of Different Types of Organic Load}

We examined the effect of 3 commonly used simulated organic materials $(5 \%$ and $10 \%$ fetal calf serum and ASTM E2197 standard organic load consisting of 5\% tryptone, $0.4 \%$ mucin, and $5 \%$ bovine serum albumin) on killing of MRSA. ${ }^{18}$ The $10-\mu \mathrm{L}$ inoculum of MRSA prepared in each of the test organic materials was spread to cover $22-\mathrm{mm}^{2}$ steel disk carriers or allowed to dry after placement as a central droplet $\left(\sim 4-\mathrm{mm}^{2}\right.$ area). Carriers were exposed to 3 minutes of UV-C.

\section{Effect of Carrier Type}

We examined reduction in the pathogens inoculated onto steel disks, plastic (Nunc), glass slides (Fisher Scientific), and Formica (Diller). For each carrier, a $10-\mu \mathrm{L}$ inoculum was spread to cover $22 \mathrm{~mm}^{2}$. The carriers were placed 4 feet from the device at a height of 4 feet and exposed to 5 minutes of UV-C. Log reductions were enumerated against untreated control carriers of the same material.

\section{Effect of Variation in Carrier Height}

We tested killing of MRSA at different heights at a distance of 6 feet from the Optimum-UV device. Steel disk carriers were placed on the floor as well as 1, 3, 4, and 6 feet above the floor and exposed to UV-C for 5 minutes.

\section{Effect of Interrupted vs Uninterrupted Dosing}

Because some manufacturers recommend running 1 cycle on each side of the bed, we examined the impact of interrupted UV-C cycles on germicidal activity against MRSA. Steel disk carriers were placed 4 feet from the device at a height of 4 feet. Reductions in MRSA were compared for 4 different exposure cycles: (1) uninterrupted 16-minute exposure; (2) 3 exposures of 5.33 minutes each with each exposure interrupted by a 1-minute pause; (3) 3 exposures of 5.33 minutes each with each exposure interrupted by a 20-minute pause; and (4) 8 exposures of 2 minutes each with each exposure interrupted by a 1-minute pause between cycles.

\section{Statistical Analysis}

A 1-way analysis of variance was performed to compare the mean log reductions. A post hoc Tukey honest significant difference test was used to test pairwise differences between group means. For each assessment of variation in test methods, a comparison group was chosen as the standard for comparison: spreading of the inoculum, reference equals $4 \mathrm{~mm}^{2}$; orientation of steel disk carriers, reference equals horizontal orientation perpendicular to the lamps; organic load, reference 
equals no organic load; carrier material, reference equals steel; height, reference equals 4 feet; and cycle interruption, reference equals uninterrupted 16-minute exposure. For statistical analysis, we used R software. ${ }^{19}$

\section{RES U L T S}

As shown in Figure 1, there were no significant differences between the 2 devices in reduction of either pathogen after exposures of 5, 10, 20, and 40 minutes. For MRSA, a greater than $3 \log$ reduction was achieved within 5 minutes. For C. difficile spores, log reductions increased with increasing exposure time, with 20 minutes of exposure required to achieve a reduction of approximately $2 \log$.

As shown in Figure 2, spreading of the inoculum over a greater surface area significantly enhanced killing of both pathogens. For $C$. difficile spores, spreading of the inoculum to cover $22 \mathrm{~mm}^{2}$ resulted in a reduction of approximately $2 \log$ within 10 minutes of exposure at 3 feet. For MRSA, spreading of the inoculum to cover $22 \mathrm{~mm}^{2}$ resulted in a reduction of approximately $5 \mathrm{log}$ within 10 minutes. Positioning of the carriers vertically in parallel with the lamp also enhanced killing of both pathogens in comparison with a perpendicular orientation (Figure 3).

Figure 4 shows the effect of different types of organic load on UV-C efficacy against MRSA. The ASTM standard organic load and $10 \%$ fetal calf serum significantly reduced killing of
MRSA when the inoculum covered a $4-\mathrm{mm}^{2}$ surface area, whereas $5 \%$ fetal calf serum did not. However, none of the organic load suspensions significantly affected killing of MRSA when the inoculum was spread to cover $22 \mathrm{~mm}^{2}$.

As shown in Figure 5, the log reductions of the pathogens achieved on plastic, Formica, and glass slides were not significantly different from the reductions on steel disks. Of note, when the $10-\mu \mathrm{L}$ inoculum was not manually spread, the reduction of pathogens was significantly greater on glass slides than on steel disks (data not shown); it was observed that natural spreading of the inoculum occurred on glass but not on the steel disk. At 6 feet from the device, there were no significant differences in log reductions of MRSA achieved at different heights ranging from floor level to 6 feet above floor level $(P=.88)$. There were also no significant differences in log reductions of MRSA with 16 minutes of UV-C exposure that was uninterrupted versus administered in interrupted doses.

\section{I SCUSSION}

In experimental testing, we found that the Tru-D and Clorox Healthcare Optimum-UV devices were equally effective for killing of MRSA and $C$. difficile spores. Using the OptimumUV device, we demonstrated that variation in experimental testing conditions can have a significant impact on the measured efficacy of UV-C. Killing of the pathogens was significantly enhanced by spreading of the inoculum over an

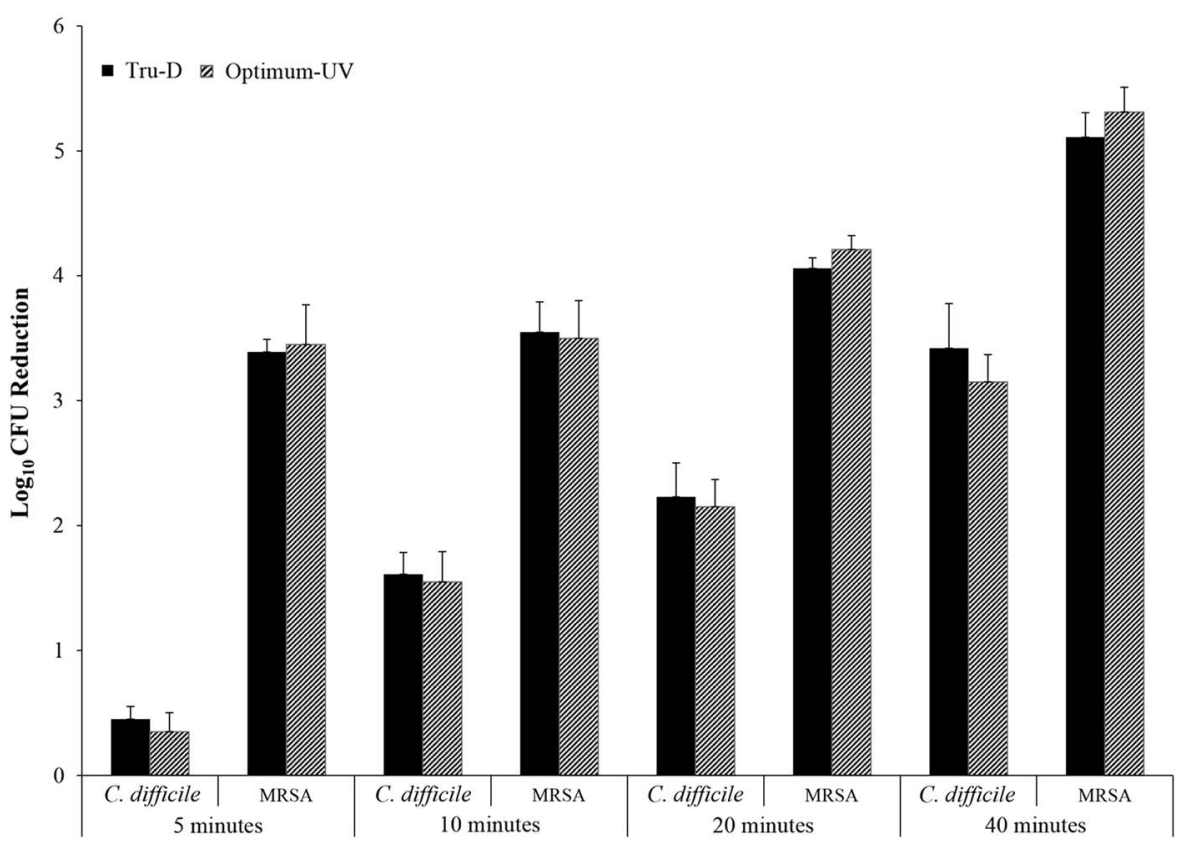

FIGURE 1. Efficacy of the Tru-D versus Clorox Healthcare Optimum-UV System for killing of Clostridium difficile spores and methicillinresistant Staphylococcus aureus (MRSA). Steel disk carriers were inoculated with $1 \times 10^{6}$ colony-forming units (CFU) of the pathogens in $10 \mu \mathrm{L}$ of phosphate-buffered saline and the inoculum was spread to cover the $10-\mathrm{mm}^{2}$ surface area of the disk. The carriers were placed 4 feet from the devices at a height of 4 feet and irradiated for 5, 10, 20, or 40 minutes. The means of data from triplicate experiments are presented. Error bars indicate standard error. 


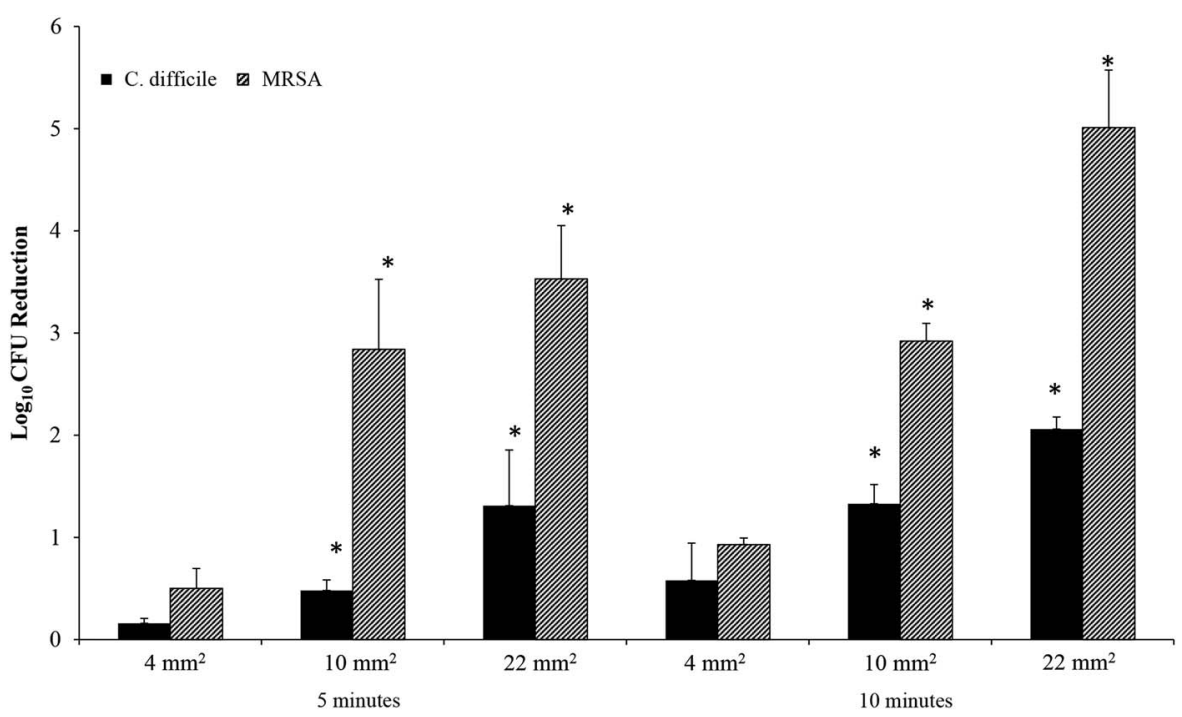

FIGURE 2. Effect of inoculum dispersal on killing of Clostridium difficile spores and methicillin-resistant Staphylococcus aureus (MRSA) by the Optimum-UV Device. Steel disk carriers were inoculated with $1 \times 10^{6}$ colony-forming units (CFU) of the pathogens in $10 \mu \mathrm{L}$ of phosphate-buffered saline and the inoculum was either not spread $\left(\sim 4-\mathrm{mm}^{2}\right.$ area on a $10-\mathrm{mm}^{2}$ disk), spread to cover the surface area of a $10-\mathrm{mm}^{2}$ disk, or spread to cover the surface area of a $22-\mathrm{mm}^{2}$ disk. The carriers were placed 4 feet from the device at a height of 4 feet and irradiated for $5,10,20$, or 40 minutes. The means of data from triplicate experiments are presented. Error bars indicate standard error. Asterisk indicates $P<.01$ in comparison with the smaller surface area.

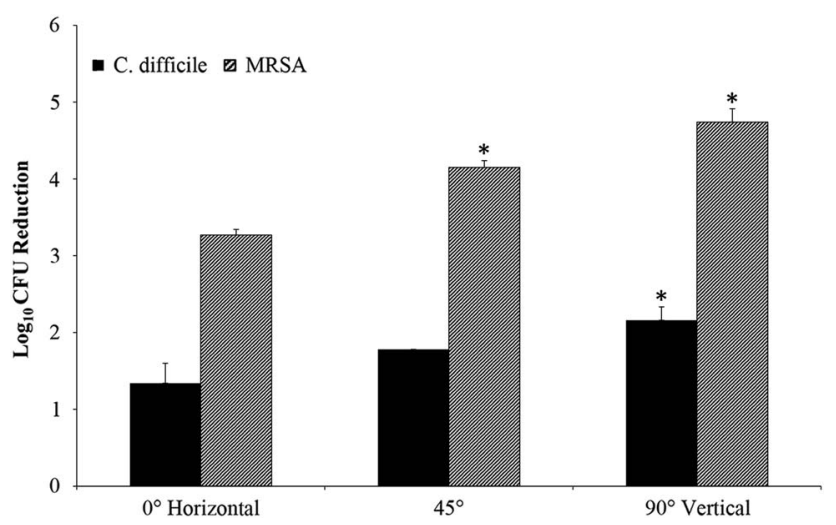

FIGURE 3. Effect of orientation of the carriers relative to the ultraviolet-C lamps on killing of Clostridium difficile spores and methicillin-resistant Staphylococcus aureus (MRSA) by the OptimumUV Device. Steel disk carriers were inoculated with $1 \times 10^{6}$ colonyforming units (CFU) of the pathogens in $10 \mu \mathrm{L}$ of phosphate-buffered saline and the inoculum was spread to cover the entire $22-\mathrm{mm}^{2}$ surface area. The carriers were adhered to glass slides and positioned in parallel with the vertical lamp (ie, $90^{\circ}$ vertical and directly facing the lamp), perpendicular to the lamp (ie, horizontal), or at a $45^{\circ}$ angle from the lamp. The carriers were placed 4 feet from the device at a height of 4 feet and irradiated for 10 minutes. The means of data from triplicate experiments are presented. Error bars indicate standard error. Asterisk indicates $P<.01$ in comparison with the horizontal carriers.

increased surface area and orientation of carriers in parallel rather than perpendicular with the UV-C lamps. The choice of organic load also impacted the measured efficacy of UV-C.

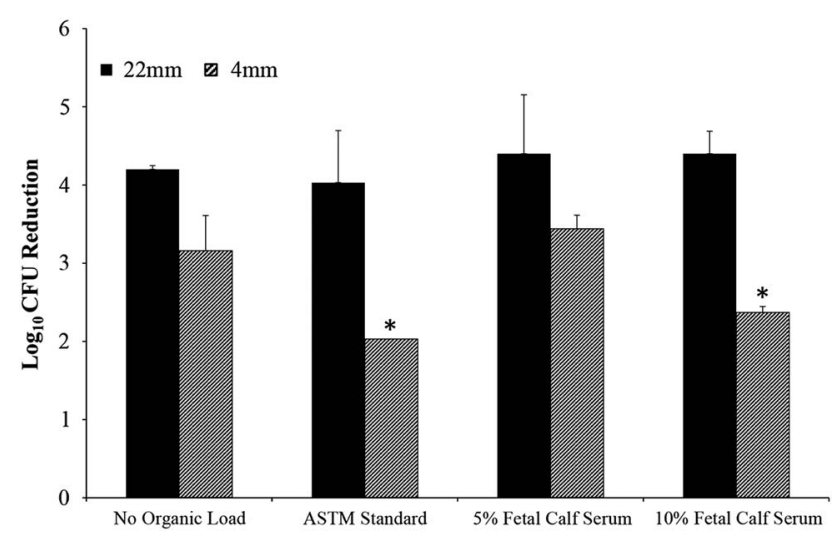

FIGURE 4. Effect of different types of organic load on killing of methicillin-resistant Staphylococcus aureus (MRSA) by the Optimum-UV Device. Steel disk carriers were inoculated with $1 \times 10^{6}$ colony-forming units (CFU) of MRSA in $10 \mu \mathrm{L}$ of the organic load solution and the inoculum was either allowed to dry as a central droplet $\left(\sim 4-\mathrm{mm}^{2}\right.$ area $)$ or spread to cover the entire $22-\mathrm{mm}^{2}$ surface area. The carriers were placed 4 feet from the device at a height of 4 feet and irradiated for 3 minutes. The means of data from triplicate experiments are presented. Error bars indicate standard error. Asterisk indicates $P<.01$ in comparison with reductions in the absence of organic load.

Our findings highlight the need for development of standardized methods for testing the efficacy of UV-C devices. Such standardization is needed to facilitate comparative 


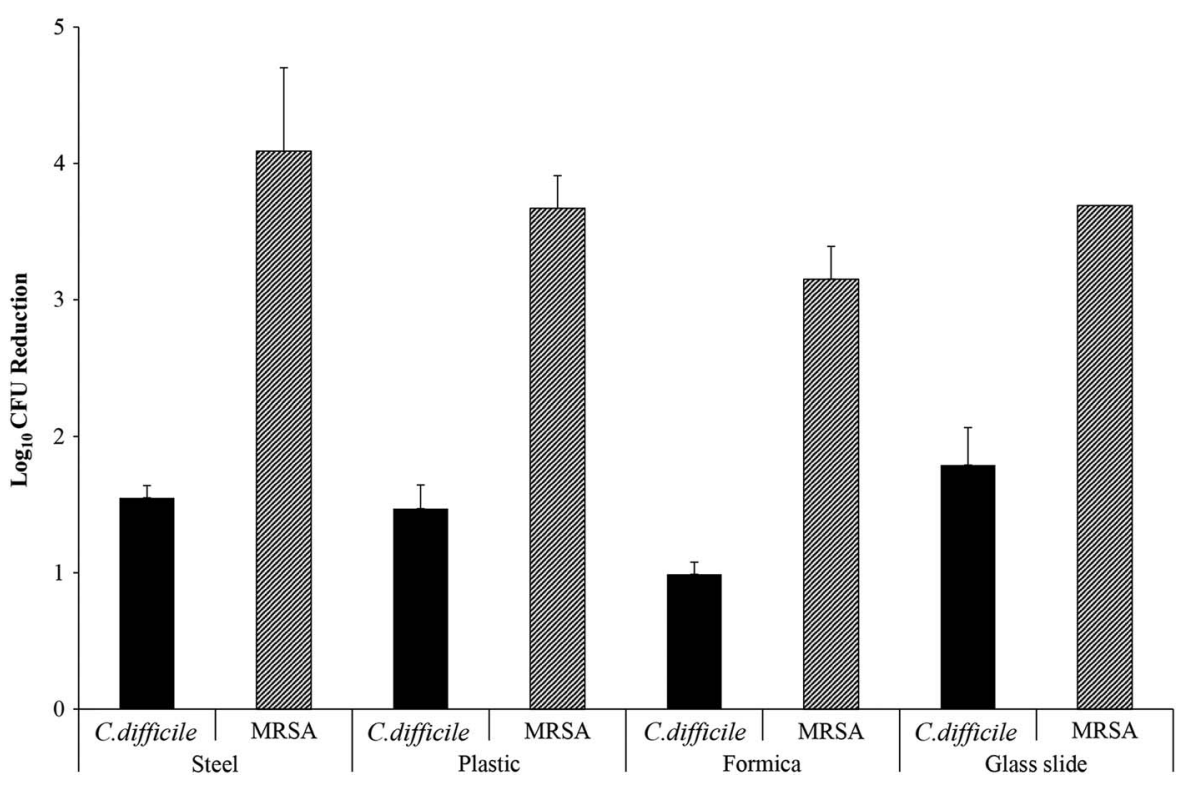

FIGURE 5. Effect of different types of carriers on killing of Clostridium difficile spores and methicillin-resistant Staphylococcus aureus (MRSA) by the Optimum-UV Device. Carriers were inoculated with $1 \times 10^{6}$ colony-forming units (CFU) of the pathogens in $10 \mu \mathrm{L}$ of phosphate-buffered saline and spread to cover the entire $10-\mathrm{mm}^{2}$ surface area. The carriers were placed 4 feet from the device at a height of 4 feet and irradiated for 5 minutes. The means of data from triplicate experiments are presented. Error bars indicate standard error.

effectiveness evaluations, particularly since there may be significant differences in performance of different UV-C devices. ${ }^{8}$

The most important practical implication of our results is that relatively short UV-C cycles could potentially be effective in reducing pathogens, including $C$. difficile spores, on surfaces. We found that 20 minutes was required to achieve a $2 \mathrm{log}$ reduction in C. difficile spores spread to cover $10 \mathrm{~mm}^{2}$, whereas only 10 minutes was required to achieve a similar reduction in spores spread to cover $22 \mathrm{~mm}^{2}$. Similarly, Rutala et $\mathrm{al}^{10}$ found that a 10-minute UV-C exposure was sufficient to reduce C. difficile spores by approximately $3 \log$ when the spore inoculum was spread to cover a Rodac template $\left(\sim 25 \mathrm{~cm}^{2}\right)$. Such cycle times would be much shorter than the approximately 45-minute cycles currently recommended for the Tru-D when used in rooms of patients with $C$. difficile infection. ${ }^{3-4}$ Given that pathogens are typically present in relatively low concentrations on hospital surfaces, ${ }^{3,8}$ further studies are indicated to validate the effectiveness of relatively short UV-C exposure times in reducing the burden of C. difficile spores in isolation rooms.

Our findings have some additional practical implications. Since organic load may reduce efficacy of UV-C, our findings support the recommendation that surfaces be cleaned prior to UV-C exposure. ${ }^{4,9}$ The finding that interrupted and uninterrupted UV-C exposures were similarly effective suggests that the practice of running 2 cycles in different locations in the room will not adversely affect performance. ${ }^{1-12}$ Finally, positioning of objects such as call buttons or tables in close proximity to UV-C devices has been recommended to take advantage of the fact that efficacy is reduced as distance from the device increases. ${ }^{3-8}$ Our results suggest that orientation of objects such that the largest surface area is in parallel with the UV-C lamps may enhance efficacy.

Our study has some limitations. We evaluated only 2 pathogens and tested only 1 strain of each. Moore et $\mathrm{al}^{20}$ reported that spores of an epidemic ribotype 027 strain were less susceptible to UV-C than a ribotype 001 strain. Similarly, our group reported some variability in killing of different strains of $C$. difficile and vancomycin-resistant Enterococcus by UV-C. ${ }^{3}$ To standardize testing of chemical disinfectants against $C$. difficile spores, the Environmental Protection Agency has recommended that 1 clinically relevant strain (ATCC 43598) be used and that a standard method be used for spore preparation. ${ }^{21}$ The C. difficile strain used in our study was ATCC 43598. Additional studies are needed to assess the impact of different methods of preparation and handling of $C$. difficile spores because factors such as triggering of germination or heat activation could have a significant impact on UV-C efficacy. ${ }^{22-23}$

\section{ACKNOWLEDGMENTS}

Financial support: Department of Veterans Affairs (Merit Review grant to C.J.D.); and Clorox. Clorox provided a Clorox Healthcare Optimum-UV System to be used for the study. Clorox did not provide any input on data analysis or writing and editing of the manuscript.

Potential conflicts of interest: C.J.D. reports that he has served on advisory boards for Clorox and 3M and has received research grants from 3M, EcoLab, GOJO, and Clorox. All other authors report no conflicts of interest relevant to this article.

Address correspondence to Curtis J. Donskey, MD, Geriatric Research, Education, and Clinical Center 1110W, Cleveland VA Medical Center, 10701 East Blvd, Cleveland, OH 44106 (curtisd123@yahoo.com). 


\section{REFERENCES}

1. Rutala WA, Weber DJ. The role of the environment in transmission of Clostridium difficile infection in health care facilities. Infect Control Hosp Epidemiol 2011;32:207-209.

2. Owens MU, Deal DR, Shoemaker MO, et al. High-dose ultraviolet $\mathrm{C}$ light inactivates spores of Bacillus subtilis var. niger and Bacillus anthracis Sterne on non-reflective surfaces. Appl Biosafety 2005; 10:240-247.

3. Nerandzic MM, Cadnum JL, Pultz MJ, Donskey CJ. Evaluation of an automated ultraviolet radiation device for decontamination of Clostridium difficile and other healthcare-associated pathogens in hospital rooms. BMC Infect Dis 2010;10:197.

4. Rutala WA, Gergen MF, Weber DJ. Room decontamination with UV radiation. Infect Control Hosp Epidemiol 2010;3110:1025-1029.

5. Nerandzic MM, Fisher CW, Donskey CJ. Sorting through the wealth of options: comparative evaluation of two ultraviolet disinfection systems. PLoS One 2014;23(9):e107444.

6. Boyce JM, Havill NL, Moore BA. Terminal decontamination of patient rooms using an automated mobile UV light unit. Infect Control Hosp Epidemiol 2011;32:737-742.

7. Havill NL, Moore BA, Boyce JM. Comparison of the microbiological efficacy of hydrogen peroxide vapor and ultraviolet light processes for room decontamination. Infect Control Hosp Epidemiol 2012;33:507-512.

8. Nerandzic MM, Thota P, Sankar CT, et al. Evaluation of a pulsed xenon ultraviolet disinfection system for reduction of healthcareassociated pathogens in hospital rooms. Infect Control Hosp Epidemiol 2015;36:192-197.

9. Zhang A, Nerandzic MM, Kundrapu S, Donskey CJ. Does organic material on hospital surfaces reduce the effectiveness of hypochlorite and UV radiation for disinfection of Clostridium difficile? Infect Control Hosp Epidemiol 2013;34:1106-1108.

10. Rutala WA, Gergen MF, Tande BM, Weber DJ. Room decontamination using an ultraviolet-C device with short ultraviolet exposure time. Infect Control Hosp Epidemiol 2014;35:1070-1072.

11. Jinadatha C, Quezada R, Huber TW, Williams JB, Zeber JE, Copeland LA. Evaluation of a pulsed-xenon ultraviolet room disinfection device for impact on contamination levels of methicillin-resistant Staphylococcus aureus. BMC Infect Dis 2014; 14:187.

12. Stibich M, Stachowiak J, Tanner B, et al. Evaluation of a pulsedxenon ultraviolet room disinfection device for impact on hospital operations and microbial reduction. Infect Control Hosp Epidemiol 2011;32:286-288.

13. Anderson DJ, Gergen MF, Smathers E, et al. Decontamination of targeted pathogens from patient rooms using an automated ultraviolet-C-emitting device. Infect Control Hosp Epidemiol 2013;34:466-471.

14. Levin J, Riley LS, Parrish C, English D, Ahn S. The effect of portable pulsed xenon ultraviolet light after terminal cleaning on hospital-associated Clostridium difficile infection in a community hospital. Am J Infect Control 2013;41:746-748.

15. Haas JP, Menz J, Dusza S, Montecalvo MA. Implementation and impact of ultraviolet environmental disinfection in an acute care setting. Am J Infect Control 2014;42:586-590.

16. Simmons S, Morgan M, Hopkins T, Helsabeck K, Stachowiak J, Stibich M. Impact of a multi-hospital intervention utilising screening, hand hygiene education and pulsed xenon ultraviolet (PX-UV) on the rate of hospital associated methicillin resistant Staphylococcus aureus infection. J Infect Prevent 2013;14: $1-3$.

17. ASTM International. Designation E2197: standard quantitative disk carrier test method for determining bactericidal, virucidal, fungicidal, mycobactericidal, and sporicidal activities of chemicals. West Conshohocken, PA: ASTM, 2011.

18. Sorg JA, Sonenshein AL. Bile salts and glycine as cogerminants for Clostridium difficile spores. J Bacteriol 2008;190:2505-2512.

19. R Development Core Team. R: a language and environment for statistical computing. R project website. http://www.R-project. org/. Published 2012. Accessed January 9, 2016.

20. Moore G, Ali S, Cloutman-Green EA, et al. Use of UV-C radiation to disinfect non-critical patient care items: a laboratory assessment of the Nanoclave Cabinet. BMC Infect Dis 2012; $12: 174$.

21. Guidance for the efficacy evaluation of products with sporicidal claims against Clostridium difficile (June 2014). US Environmental Protection Agency website. http://www.epa. gov/pesticide-registration/guidance-efficacy-evaluation-productssporicidal-claims-against-clostridium. Accessed January 1, 2015.

22. Nerandzic MM, Donskey CJ. Triggering germination represents a novel strategy to enhance killing of Clostridium difficile spores. PLoS One 2010;5(8):e12285.

23. Nerandzic MM, Donskey CJ. Activate to eradicate: inhibition of Clostridium difficile spore outgrowth by the synergistic effects of osmotic activation and nisin. PLoS One 2013;8:e54740. 